\title{
The forces behind fin regeneration
}

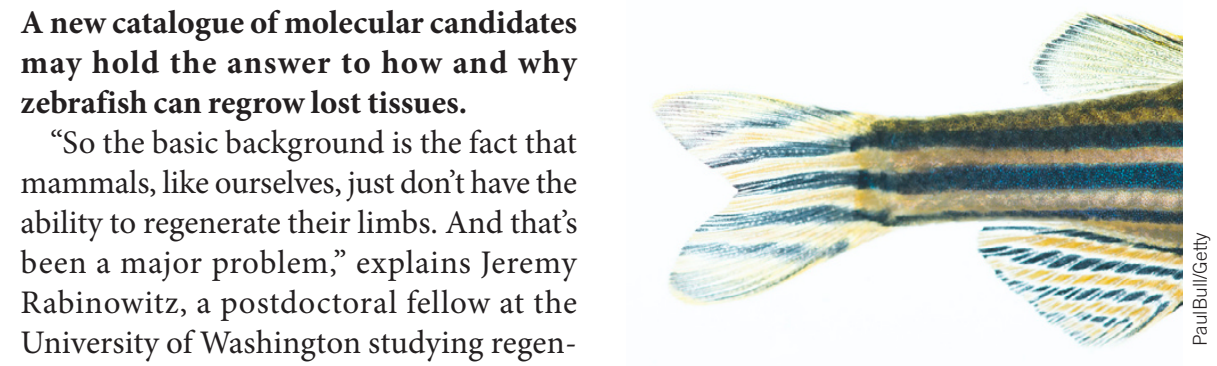
eration and homeostatic growth. In some cases, a lost fingertip may grow back, but we aren't so lucky after the loss of larger structures. Some non-mammalian species, however, don't have this same limitation. The salamander, with its anatomical similarities to humans, has been the classic model for studying limb regeneration, but knock the fin off of a zebrafish and it grows back just as well, able to replace lost tissue to the exact size and structure that it was before the amputating injury.

The reason for this remarkable ability is thought to be thanks to gradients of molecules that form what is known as positional memory, a property first depicted over 100 years ago by Thomas Morgan. This memory, Rabinowitz explains, allows the remaining cells after an injury to figure out how much tissue was lost and how much they need to regenerate to get back to normal. In salamanders, Prod1, a transmembrane receptor protein that is expressed in a gradient along uninjured limbs, is the only molecule known that can regulate positional memory. No such target has been identified for zebrafish yet, but Rabinowitz and his colleagues have recently produced a catalogue of candidates to help narrow down the search (Proc. Natl. Acad. Sci. USA, 114, E717-E726; 2017).
Previous regeneration work has focused on gene expression changes that occur after injury, but Rabinowitz took a different approach, focusing instead on the expression of different molecules in standing adult tissues. "What I wanted to do was just use different technologies that we have right now to map potential gradients that exist in the uninjured tissues." Using RNAseq and proteomic and metabolomics approaches, he and his colleagues mapped a straight line across the intact caudal fin of their zebrafish, divided it into proximal, middle, and distal regions, and measured what molecules-whether transcripts, proteins, or metabolites-were enriched in one direction or the other. From hundreds of potential molecules, they filtered down to a high-confidence list of 32 genes and 42 metabolites, presented in the paper and its accompanying data sets, that they believe are strong candidates for controlling positional memory.

With that list of candidates in hand, they next wanted to see which of those were maintained during the earliest stages of regenerating an injured fin. "I think that was a pretty vital way to look at it, because if it's different before injury but then immediately changes, it's probably unlikely that it's really contributing to positional memory," he explains. From that follow-up comparison, a short list emerged. At the top are three genes in particular-aldh1l1, $h s d 17 b 7$, and muc5.2-that were differentially expressed in both uninjured limbs and along the regrowing tissue. Given the ease of manipulating gene expression and pathways in zebrafish, he plans on pursuing those candidates with transgenic animals, hoping to create inducible systems across the whole fish as well as within specific structures to see just how these different molecules impact regenerative ability.

Although approaching future work from the angle of regeneration, Rabinowitz hopes that this catalogue will help others answer questions about adult tissue homeostasis more broadly, an area he remarks where "there's not a tremendous amount known about how structures maintain their proper size in adult stages." Several candidates identified in the study, particularly some of the transcription factors, he explains, have established roles during zebrafish development; how and why they may still be needed for tissue maintenance in adults is an exciting future question.

Will any of these molecules someday let us regenerate an entire lost limb? Short answer, says Rabinowitz, it is much too early to tell. But probing the positional memory of these candidates and determining the different pathways that they influence in zebrafish as well as salamanders and other models may one day help put us on the path to regrowing more than just our fingertips. Ellen P. Neff 